\title{
Neural Network-Control Scheme for Grid Connected Hybrid Power Generation System for Power Quality Improvement
}

\author{
N.Prakash ${ }^{1}$, K.Sankar ${ }^{2}$, \\ ${ }^{1}$ Department of EEE, Adhiyamaan College of Engineering,Hosur-635 109Tamilnadu, India. \\ ${ }^{2}$ Assistant professor, Department of EEER oever Engineering College, Perambalur-621 212Tamilnadu, \\ India
}

\begin{abstract}
A Wind-Diesel Power Generation system with neural network is proposed in this paper. The system consists of Wind Power, Diesel Engine and an Intellectual controller. In the proposed topology, the Diesel Engine and the Wind Turbine are variable-speed machines, permitting Maximum fuel efficiency and Maximum energy extract from the wind. MATLAB2009b/Simulink was used to build the dynamic model and simulate the system. The pitch angle of wind turbine is controlled by the Modified Elman Neural Network(MENN).An Modified ENN is used to control Maximum Power Point Tracking(MPPT) and to reduce the THD in the Grid.ENN is a Partial Recurrent Neural Network which is used to achieve fast and stable response for the real power control and its issues.
\end{abstract}

Key Words: Modified Elman Neural Network, Maximum Power Point Tracking, Diesel Engine, Total Harmonic Distortion, Converters.

\section{Introduction}

In recent years, the development of Wind Energy Generation has been associated with wind farms located onshore and offshore. Variable-Speed wind turbines have many advantages. The wind farms are connected to strong transmission grids and their power ranges from some tens to a hundred megawatts[5].

Wind turbines can operate with Maximum Aerodynamic Efficiency, and the power fluctuations can be absorbed as an inertial energy in the blades [1]. In some applications, the wind turbine may be augmented by an additional source, and usually a Diesel Generator. These systems are called Wind-Diesel systems $[5,1]$ and they may be used to supply electrical energy to stand-alone loads, e.g., small villages that are not connected to the main utility. Most Diesel Generation systems operate at a constant rotational speed due to the restriction of constant frequency at the Generator terminals. However, Diesel engines have high fuel consumption when operating at light loads and constant speed [12]. More-over, for light loads at rated speed operation, not all the fuel is burned by the engine and wet stacking is produced. This increases maintenance costs [8]. In order to improve the efficiency and avoid wet stacking, a minimum load of about $30 \%$ to $40 \%$ is usually recommended by the manufacturers.

The combination of battery energy storage and Wind Generating System in distributed power system can provide the effective, reliable, and durable power system. The system also provides energy saving and un-interruptible power within distribution network[10]. The parallel processing of wind energy generating system and battery storage will enhance the power flow in the distributed network. The wind energy generating system is used to charge the battery as and when the wind power is- available. The control method for the state of charge of battery unit was proposed in [10,2]. The battery storage provides a rapid response for either charging/discharging the battery and also acts as a constant voltage source for the critical load in the distributed network.

Li et al [6], proposed a small Wind Generation System with Neural Network for wind-speed estimation and PI control for maximum wind-power extraction. The mechanical power of the wind turbine can be well tracked for both the dynamic and steady state, but the power deviation and speed tracking errors are large for transient response lasting for almost 20 secs.

Muljadi et al[3] developed pitch control and generator load control methods to adjust the aerodynamic power, but power coefficient $C_{p}$ deviation is too large [3]. In order to improve the ability of identifying high-order systems, some modified ENN have been proposed recently, which proved to have more advantages than the basic ENN, including a better performance, higher accuracy, dynamic robustness, and a fast transient performance.

The Elman neural network (ENN) is a partial recurrent network model first proposed by Elman [4] which is a superior network. The ENN does not need to use the state as input or training signal, which makes the ENN superior to static feed-forward network and is used in dynamic system identifications widely [11]. 


\section{System Description}

Recently, the growth of wind power generation systems has exceeded the most optimistic estimation [11]. In this paper, a stand-alone hybrid energy system consisting of wind and diesel is proposed with the battery for energy storage. Wind is the primary power source of the system to take full advantage of renewable energy; diesel may be used as a backup system.

The proposed Diesel and Wind Hybrid system is shown in Fig 1.

\subsection{Wind Power Generation System (WPGS)}

In order to achieve the maximum power point in the wind power generation system, it is necessary to install the Power Electronic Converters between the Wind Turbine Generator (WTG) and the Grid. The function of the wind turbine is to convert kinetic energy in to mechanical energy

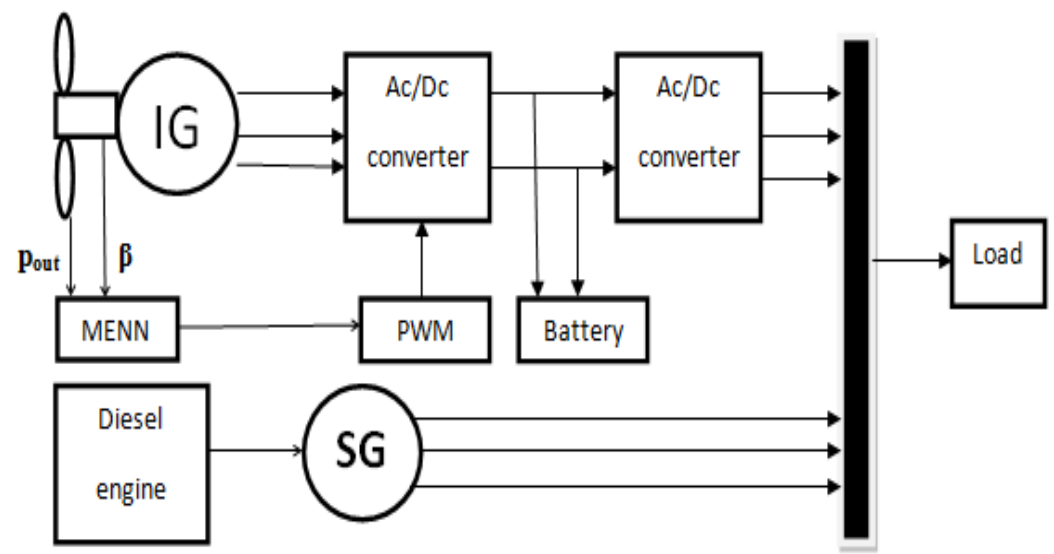

Fig 1: Proposed Block Diagram

Which is given to the generator. The mechanical power output of Generator is expressed as

$$
P_{m}=\frac{1}{2} \rho A V_{\omega}^{2} C_{p}(\beta, \lambda)
$$

Where $\rho$ is the air density, A is the area swept by blades, $V \omega$ is the wind velocity in $\mathrm{m} / \mathrm{s}$, and $C p$ is power coefficient. It is the function of blade pitch angle $\beta$ and tip speed ratio $\lambda$. Tip speed ratio is defined by

$$
\lambda=\frac{w_{r}}{V_{w}}
$$

Where $r$ is the wind turbine blade radius, and $\omega_{r}$ is the turbine speed. A variable-speed pitchregulated wind turbine is considered in this paper, where the pitch angle controller plays an important role. Fig. 2. Shows the groups of $C p-\lambda$ curves of the wind turbine used in this study at different pitch angles [9]. From the figure that $C p$ can be controlled by varying the pitch angle $\beta$. So the output power of the wind turbine can be adjusted by pitch angle control.

\subsection{Diesel Engine System}

The Diesel-generator Set (DGS) model comprises of combustion Chamber, Drive Train, and Synchronous Generator Models. A common Governor Model is used in this paper; the essential features can be described by the transfer function described in [1].

The fuel consumption of a diesel engine depends on the speed and torque of the machine. Fig. 3 shows the fuel consumption curves of a diesel engine for various rotational speeds. It can be seen that at $20 \%$ rated power, there is $50 \%$ fuel saving than that at 0.6 rated speed. According to 


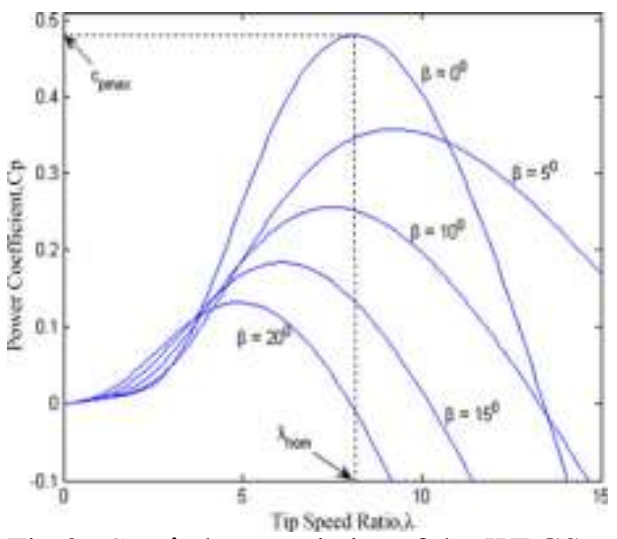

Fig 2: $C p-\lambda$ characteristics of the WPGS At different pitch angles.

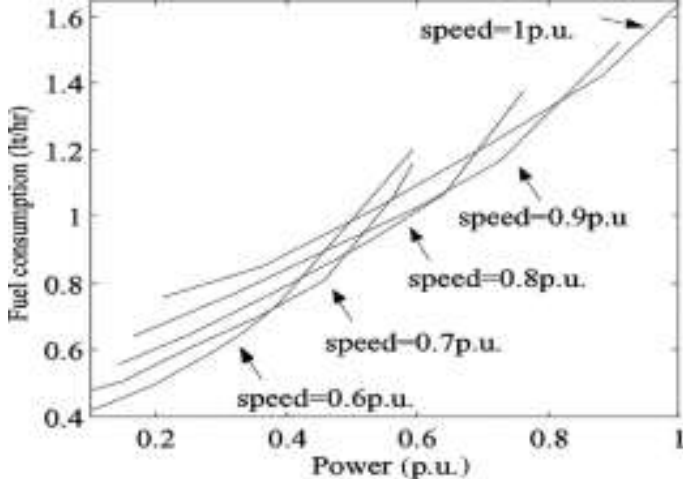

Fig 3: Fuel consumption Vs Power at Various rotational speeds.

Fig. 3, a continuous function for the optimal operation Vs various speed can be formed tangent to all the curves. In order to minimize the fuel consumption, the speed demand (optimum speed) for the diesel engine is calculated by building up a look-up table where the optimal power-speed curve is implemented.

\subsection{Battery Energy Storage System (BESS)}

The battery load current rapidly changes according to changes in weather conditions and power command for the bus inverter in operation. The DC-bus voltage will be regulated to stay within a stable region regardless of the battery-current variation. When the DC bus voltage $V_{\mathrm{dc}}$ becomes larger than the upper limit $V_{\mathrm{dc}}$ up, charging mode begins with the voltage command $V^{*}{ }_{\mathrm{dc}}$ equal to the upper limit and continues until the DC voltage reaches the limit. If $V_{\mathrm{dc}}$ goes below the lower limit $V_{\mathrm{dc}}$ low , then the voltage target is bounded at the lower limit and the converter starts operating in boost mode.

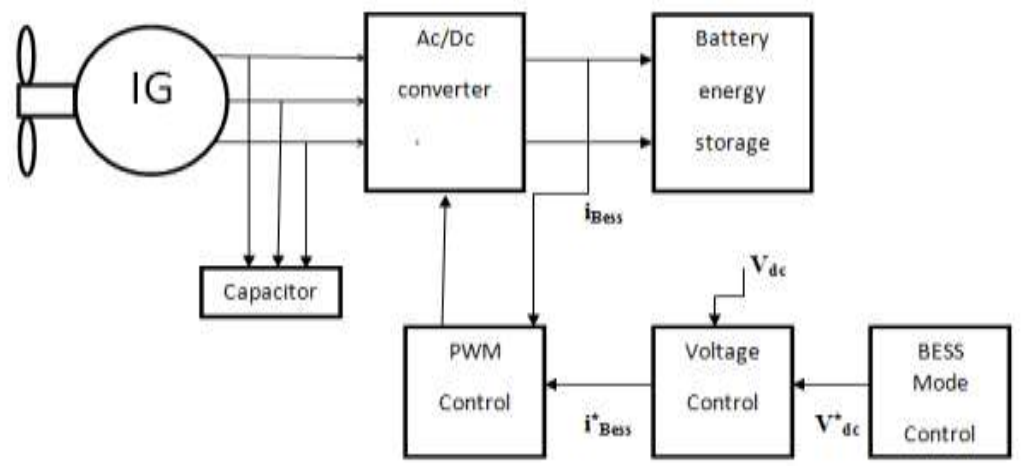

Fig 4: BESS structure with Interconnection diagram

\section{Mppt Control Algorithm For Wpgs}

\subsection{Design of Modified Elman Neural Network}

Architecture of an ENN including the input layer, the hidden layer, the context layer, and output layer which is shown in Fig.5, Modified ENN also similar to ENN which has the major difference multiplied by its coefficient $\alpha$ ranging as $(0 \leq \alpha<1)$ shown in fig .5, In proposed system the MENN has two inputs where the control law is defined as $i_{q}^{*}$, and the ENN inputs are $e^{(1)}{ }_{1}$ and $e^{(1)}{ }_{2}$ with, $e^{(1)}{ }_{1}=P_{\text {ref }}-P_{\text {out }}$ and $e^{(1)}=\beta_{c}-\beta$ in this paper.

The proposed MENN [4,7] takes the feedback into account, and a better learning efficiency can be obtained. Moreover, to make the neurons sensitive to the history of input data, self connections

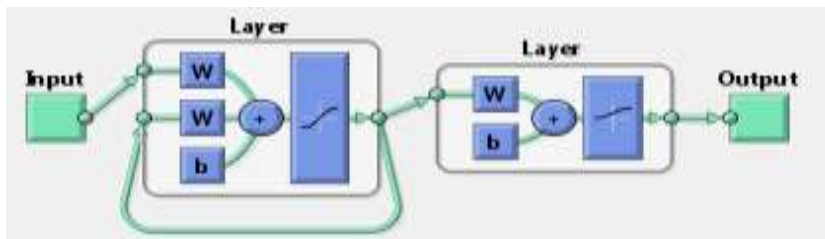

Fig4: Simple Elman Neural Network. 
Of the context nodes and output feedback node are added. So, the proposed MENN combines the ability of dealing with nonlinear problems, can effectively improve the convergence precision and reduce learning time.

The basic functions of each layer are as follow

\section{Layer 1: Input Layer}

In the input layer, the node is defined by

$$
\begin{aligned}
\operatorname{net}^{(1)} & =e^{(1)}{ }_{i}(k) \\
x^{(1)}{ }_{i}(k) & =f^{(1)}{ }_{i}\left(\operatorname{net}^{(1)}{ }_{i}(k)\right)=\operatorname{net}^{(1)}
\end{aligned}
$$

Where $k$ represents the $k$ th iteration, $e^{(1)}{ }_{i}(k)$ and $x^{(1)}{ }_{i}(k)$ are the input and the output of the layer, respectively.

\section{Layer 2: Hidden Layer}

In the hidden layer, the node is defined by

$$
\begin{aligned}
& \operatorname{net}_{j}^{(2)}=\sum_{i}\left(W_{i j} \times x^{(1)}{ }_{i}(k)\right)+\sum_{r}\left(W_{r j} \times x_{r}^{(3)}(k)\right)(5) \\
& x_{j}^{(2)}(k)=1 / 1+\exp \left(-\operatorname{net}^{(2)}{ }_{j}\right)
\end{aligned}
$$

Where $x_{i}^{(1)}$ and $x_{r}^{(3)}$ are input and $x_{j}^{(2)}(k)$ is output of the hidden layer. $x_{r}^{(3)}(k)$ is also the output of the context layer, and $W_{i j}$ and $W_{r j}$ are the connecting weights of input neurons to hidden neurons and context neurons to hidden neurons, respectively.

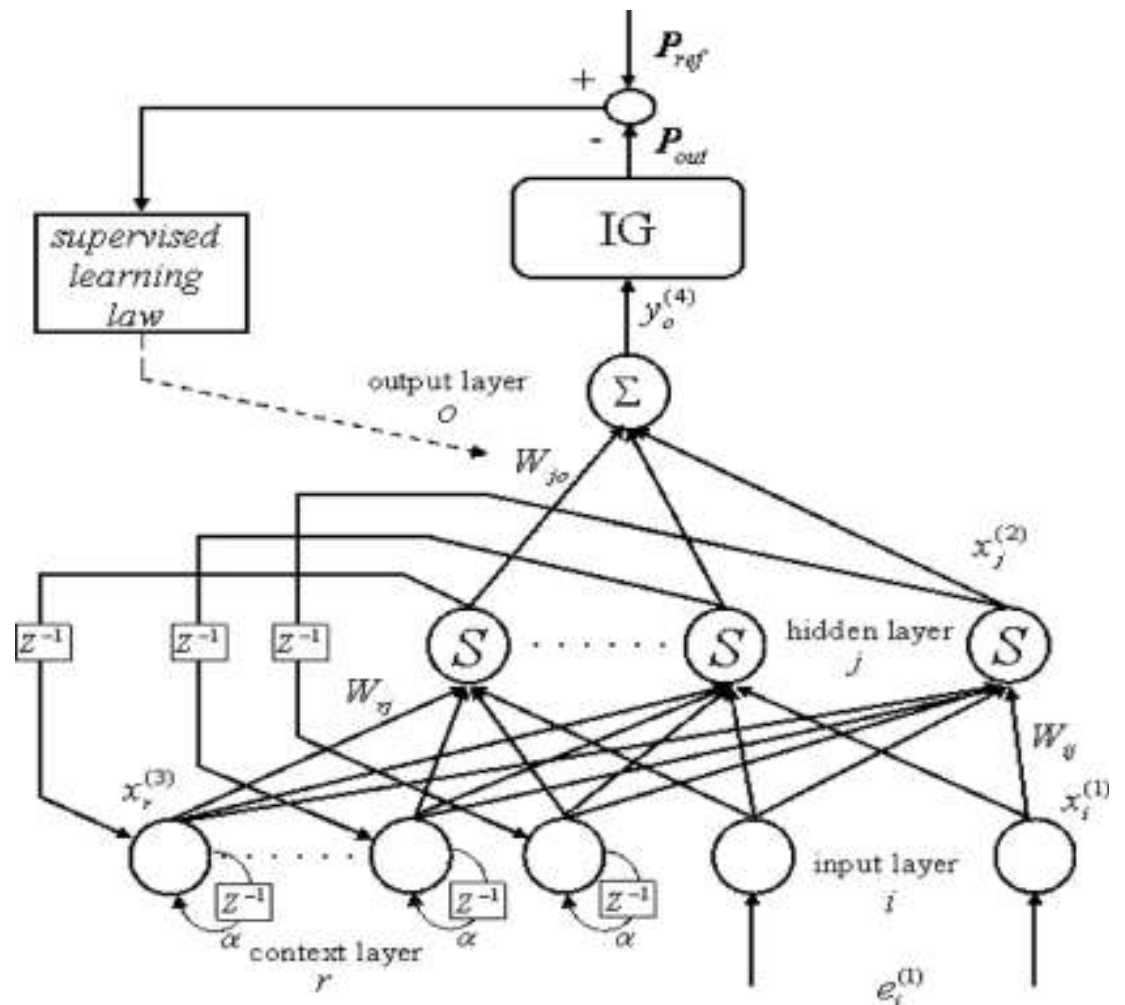

Fig 5: Structure of Modified Elman Neural Network

\section{Layer 3: Context Layer}

In the context layer, the node input and output are represented as,

$$
x_{r}^{(3)}(k)=\alpha x_{r}^{(3)}(k-1)+x_{j}^{(2)}(k-1)
$$

Where $0 \leq \alpha<1$ is the self-connecting feedback gain. 


\section{Layer 4: Output Layer}

In the output layer, the node input and output are represented as

$$
\begin{aligned}
& \operatorname{net}^{(4)}{ }_{o}(k)=\Sigma_{j} W_{j o} \times x^{(2)}{ }_{j}(k) \\
& y^{(4)}{ }_{o}(k)=f^{(4)}{ }_{o}\left(\operatorname{net}^{(4)}{ }_{o}(k)\right)=\operatorname{net}^{(4)}{ }_{o}(k)=i^{*}{ }_{q}
\end{aligned}
$$

Where $W_{j o}$ is the connecting weight of hidden neurons to output neurons, and $y^{(4)}{ }_{o}(k)$ is the output of the MENN and also the control effort of the proposed controller.

Once the MENN has been initialized, a supervised learning is used to train this system based on gradient descent. The derivation is the same as that of the back-propagation algorithm [4,7]. It is employed to adjust the parameters $W_{j o}, W_{r j}$, and $W_{i j}$ of the MENN by using the training patterns. By recursive application of the chain rule, the error term for each layer is calculated, and updated. The purpose of supervised learning is to minimize the error function $E$ expressed as

$$
E=1 / 2\left(P_{\text {out }}-P_{\text {ref }}\right)^{2}=1 / 2 e^{2}
$$

Where $P_{\text {out }}$ and $P_{\text {ref }}$ represent the actual output power and the reference output power of the generator, respectively, and $e$ denotes the tracking error. A common supervised training algorithm is used in this paper, the essential features can be seen in[4] and [7].

\subsection{Harmonic Analysis}

Harmonic currents makes main issues in grid part. It poses a challenge in the measurement of power quality. It requires great accuracy, even for higher frequencies, since the measurement refers to interharmonics that are in the range of $0.1 \%$ of the rated current. In the proposed system the Total Harmonic Distortion (THD) is measured by the Matlab/Simulink. It should be generally less than $1.48 \%$ in the grid.

With regard to high-order harmonics, EN 50160 standard does not specify any limits but states that high-order harmonics are usually negligible, though fairly unpredictable. Therefore THD $=4.61 \%$ for nominal phase-to-phase voltage.

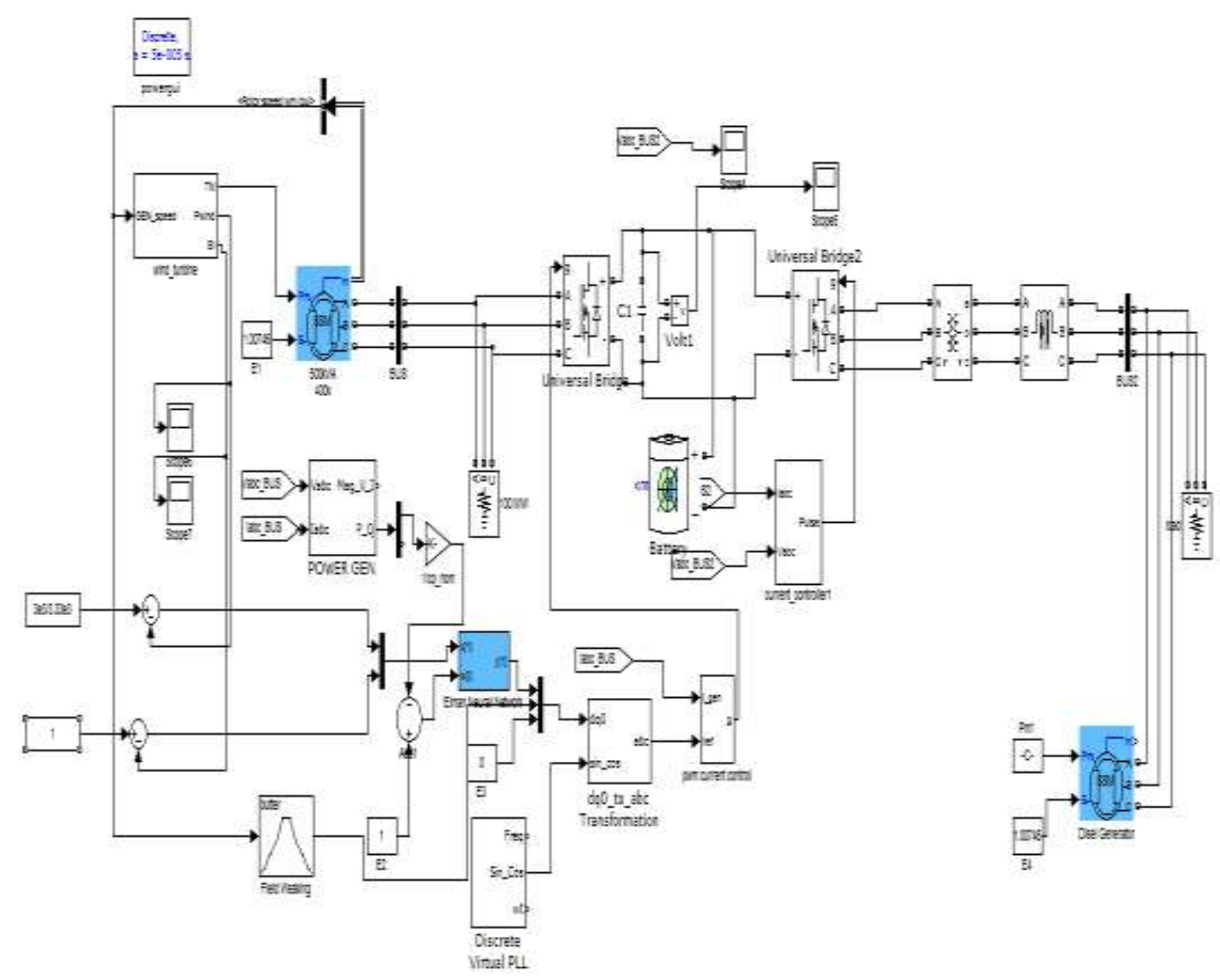

Fig 6: Simulation Diagram for Hybrid Power Generation System 


\section{Simulation Diagram And Results}

The proposed system comprises of an 3 phase Induction Generator, a current control PWM Ac/Dc converter, a Field-Orientation Mechanism including the Coordinate Translator, a Current Controlled Dc/Ac inverter, and the MPPT controller, where the ENN were applied in this paper. The Dc-bus voltage is regulated at a constant value so the real power from the wind turbine will pass to the grid. By using the Reference Frame Theory and the linearization technique, the field-oriented induction generator system can be reasonably represented by the Matlab 2009/Simulink as shown in Fig. 6 .

\section{Simulation Parameters}

Table 1: Wind Induction Generator

\begin{tabular}{|c|c|}
\hline Rated Power (KW) & 5 \\
\hline Voltage (V) & 440 \\
\hline Frequency (HZ) & 50 \\
\hline Inertia & 0.6745 \\
\hline No of poles & 4 \\
\hline Wind speed (M/S) & 12 \\
\hline
\end{tabular}

Table 2: Diesel Synchronous Generator

\begin{tabular}{|c|c|}
\hline Rated power $(\mathrm{KW})$ & 24 \\
\hline Voltage $(\mathrm{V})$ & 440 \\
\hline Frequency $(\mathrm{HZ})$ & 50 \\
\hline Inertia & 0.75 \\
\hline No of poles & 4 \\
\hline
\end{tabular}

TABLE 3: Battery Energy Storage System \& Load

\begin{tabular}{|c|c|}
\hline Voltage (V) & 220 \\
\hline Capacity (KWH) & 15 \\
\hline Load (KW & 40 \\
\hline
\end{tabular}

Table 4: Grid

\begin{tabular}{|c|c|}
\hline \multicolumn{2}{|c|}{ Rove } \\
\hline Voltage (V) & 440 \\
\hline Frequency (HZ) & 50 \\
\hline Phase & 3 \\
\hline
\end{tabular}

The above Table 1, 2, 3 and 4 represents the Wind Induction Generator Parameters, Diesel Synchronous Generator Parameters, Battery Energy Storage Parameters, Load and Grid Parameters.

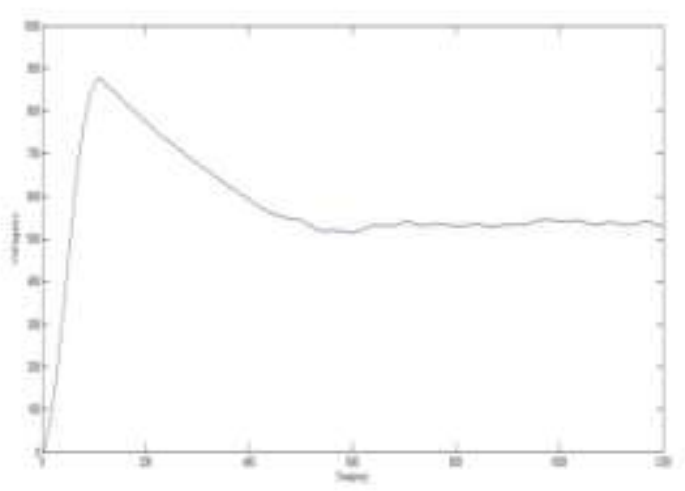

Fig 7: MPPT Tracking Response of WPG

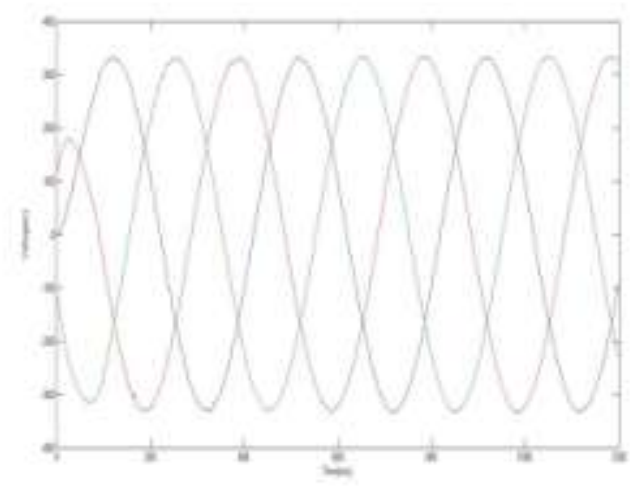

Fig 8: Grid Voltage under Sudden Load changes 


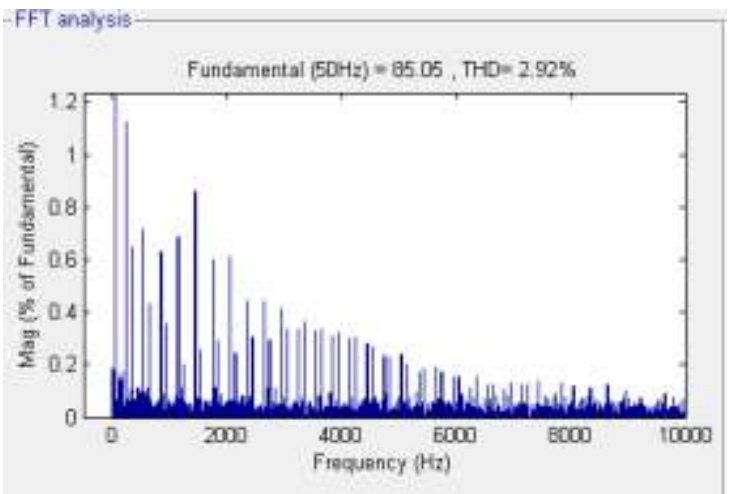

Fig 9: FFT Analysis of Grid with PI controller based STATCOM

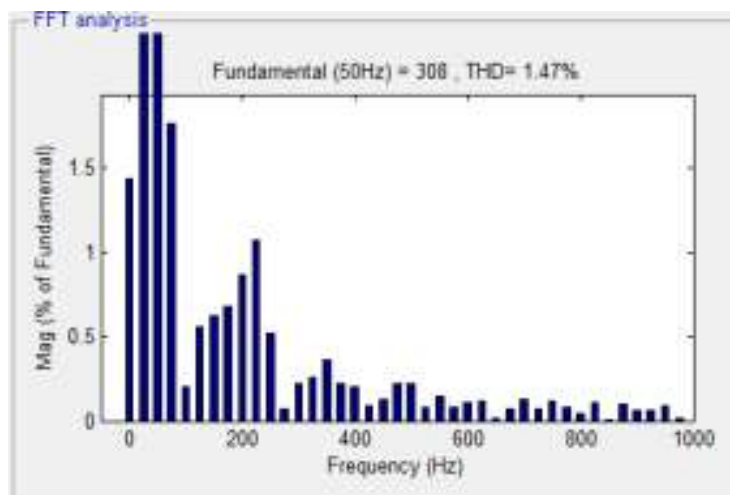

Fig 10: FFT Analysis of Grid with MENN

Figure 7 and 8 shows the output when the MPPT is achieved under WPG and the constant Grid voltage output. Figure 10 shows the output compared with PI controller based STATCOM with THD 2.92\% and in this proposed MENN controller THD is reduced to $1.47 \%$ which gives better connected to load[13].

\section{Conclusion}

In this paper, Diesel-Wind Hybrid Generation System was proposed and implemented. This StandAlone Hybrid Generation System can effectively extract the Maximum Power from the wind and maintains THD below $1.48 \%$ in grid. An efficient power sharing technique among energy sources are successfully demonstrated with more efficiency, a better transient and more stability response, even under disturbance conditions. The simulation model of the hybrid system was developed using MATLAB 2009/Simulink. The load frequency is regulated by the Diesel Generator by imposing the rotor currents with the slip frequency. The electrical torque of the WPGS generator is controlled to drive the system to the rotational speed where maximum energy can be captured. Depending on the load size and the power supplied by the WPGS generator, the control system regulates the DGS rotational speed to minimize the fuel consumption.

In future, any other source of renewable energy may be utilized in Hybrid Power Generation System and also the performance may be improved by minimising the THD. The Simulations may also be carried out by using any other software such as Proteus, Multisim etc...

\section{References}

[1] A. J. Rudell, J. A. M. Bleijs, L. Freris, D. G. Infield, and G. A. Smith, "A wind diesel system with variable speed flywheel storage,"Wind Eng., vol. 17, pp. 129-145, May 1993.

[2]. B. S. Borowy and Z. M. Salameh, "Dynamic response to a stand-alone wind energy conversion system with battery energy storage to a wind gust," IEEE Trans. Energy Convers., vol. 12, no. 1, pp. 73-78, Mar. 1997.

[3]. E. Muljadi and C. P. Butterfield, "Pitch-controlled variable-speed wind turbine generation," IEEE Trans. Ind. Appl., vol. 37, no. 1, pp. 240-246, Jan./Feb. 2001.

[4]. F. J. Lin and Y. C. Hung, "FPGA based Elman neural network control system for linear ultrasonic motor," IEEE Trans. Ferr. Ultras. And Freq. Control., vol. 56, no. 1, pp. 101-113, Jan. 2009.

[5]. G. Abad, M. A. Rodriguez, G. Iwanski, and J. Poza, "Direct power control of doubly-fed-induction-generator-based wind turbine under unbalanced grid voltage," IEEE Trans. Power Electron., vol. 25, no. 2, pp. 442-452, Feb. 2010.

[6] . H. Li, K. L. Shi, and P. G. McLaren, "Neural-network-based sensor less maximum wind energy capture with compensated power coefficient," IEEE Trans. Ind. Appl., vol. 41, no. 6, pp. 548-1556, Nov./Dec. 2005

[7]. H. Liu, S. Wang, and P. Ouyang, "Fault diagnosis based on improved Elman neural network for a hydraulic servo system," in Proc. Int. Conf. 1Robot., Autom. Mechatronics, pp no 1-6, Dec. 2006.

[8]. M. Tolbet and W.A. Peterson "Gen Sets" IEEE- April - 2003

[9]. W. D. Kellogg, M. H. Nehrir, G. Venkataramanan, and V. Greez, "Generation unit sizing and cost analysis for stand-alone wind, photovoltaic, and hybrid wind/PV systems," IEEE Trans. Energy Convers., vol. 13, no. 1, pp. 70-75, Mar. 1998.

[10]. W. Sharad Mohod, Member, IEEE, and Mohan V. Aware"Micro wind power generation with battery energy storage for critical load" IEEE Systems Journal, vol. 6, no. 1, March 2012.

[11]. Y. M. Cheng, Y. C. Liu, S. C. Hung, and C. S. Cheng, "Multi-input inverter for grid-connected hybrid PV/wind power system," IEEE Trans. Power Electron., vol. 22, no. 3, pp. 1070-1076, May 2007.

[12]. Z. Chen and Y. Hu, "A hybrid generation system using variable speed wind turbines and diesel units," in Proc. IEEE Ind. Electron. Soc. Annu. Meeting Conf., pp. 2729-2734, Nov. 2003.

[13]. Sharad w. Mohod, Mohan V. Aware," A STATCOM-control scheme for grid connected wind energy system for power quality improvement", IEEE power systems journal, vol. 4, no. 3, Sept 2010, 